\title{
Dysphagia Therapy in Adults with a Tracheostomy: A Scoping Review Protocol
}

\section{Waiza Kadri ( $\nabla$ waiza.kadri.10@ucl.ac.uk)}

Northwick Park Hospital, London North West University Healthcare NHS trust https://orcid.org/00000001-9294-1729

\section{Rhiannon Halfpenny}

Great Ormond St Hosp Children: Great Ormond Street Hospital For Children NHS Foundation Trust

\section{Breege Whiten}

University College London (UCL). Language \& Speech Science Library

\section{Christina Smith}

University College London (UCL). Division of Psychology and Language Sciences.

\section{Siofra Mulkerrin}

Addenbrookes Hospital, Cambridge University Hospitals NHS Foundation Trust

\section{Method Article}

Keywords: Tracheostomy, Dysphagia, Therapy

Posted Date: June 7th, 2021

DOl: https://doi.org/10.21203/rs.3.rs-593909/v1

License: (c) (i) This work is licensed under a Creative Commons Attribution 4.0 International License.

Read Full License 


\section{Abstract \\ Background}

Swallowing impairment (dysphagia) and tracheostomy coexist. Research in this area has often provided an overview of dysphagia management as a whole, but there is limited information pertaining to specific dysphagia therapy in the tracheostomy population. The aim of this scoping review is to provide detailed exploration of the literature with regards to dysphagia therapeutic interventions in adults with a tracheostomy. The scoping review will describe current evidence and thus facilitate future discussions to guide clinical practice.

\section{Methods}

A scoping review using the Joanna Briggs Institute and Preferred Reporting Items for Systematic Reviews guideline will be used. Ten electronic databases from inception to July 2021 and grey literature will be searched. From identified texts forward and backward citation chasing will be completed. Data extraction will compose of population demographics, aetiology and dysphagia therapy (type, design, dose and intensity). Number of citations and papers included into the scoping review will be presented visually.

\section{Discussion}

The scoping review aims to expand upon the existing literature in this field. A detailed description of the evidence is required to facilitate clinical discussions and develop therapeutic protocols in a tracheostomised population. The results of this scoping review will support future research in dysphagia therapy and provide the basis for development of best practice guidelines.

\section{Background}

A tracheostomy tube provides an alternative means of respiratory ventilation and reduces the need for prolonged endotracheal intubation. A tracheostomy creates an opening within the trachea and is generally accepted as a safe procedure that provides meaningful benefits to patients which include a secure airway and facilitating pulmonary toileting (Cheung \& Napolitano, 2014). In 2014 the National Confidential Enquiry into Patient Outcome and Death (NCEPOD) estimated that in the UK approximately 12000 patients a year had a tracheostomy inserted.

Skoretz and colleagues (2020) highlight the interdependency of swallowing and respiration. The NCEPOD (2014) reported swallowing impairments were the third most common associated complication of a tracheostomy and up to $51.6 \%(n=425)$ of inpatients with a tracheostomy had a swallowing disorder. However, there is limited knowledge regarding patients in the community with a tracheostomy and long term swallowing needs. 
The medical term for swallowing disorder is oropharyngeal dysphagia. This is an impairment of motor and/or sensory components of the swallowing mechanism in which food/fluid transfers from the oral cavity into the pharynx and through the upper oesophageal sphincter (Walshe, 2019) and can lead to aspiration: food or fluid entering the airway and passing below the vocal cords often into the lungs. Healthy adults have a degree of aspiration, however persistent aspiration can lead to aspiration pneumonitis a potentially life threatening infectious process where aspirated material is colonised by pathogenic bacteria resulting in a chest infection (Marik, 2001).

In the past, the presence of a tracheostomy tube was considered to be a primary cause of an oropharyngeal dysphagia. For instance, Bonanno (1971) suggested that a tracheostomy tube resulted in tethering of the larynx and subsequently lead to aspiration. However it is now widely recognised that dysphagia is the cause of an underlying condition of which there could be multiple reasons. This is substantiated by Wallace, McGowan and Ginnelly (2016) who argued that the presence of a tracheostomy tube does not necessarily in isolation cause an oropharyngeal dysphagia, however they do recognise that it could contribute to dysphagia. Moreover, in two separate studies completed by Leder and Ross $(2000 ; 2010)$, they reported that there was no causal relationship between tracheostomy and aspiration status. It is the role of the speech and language therapist (SLT) to work closely with people with a tracheostomy to provide detailed assessment and therapy intervention of swallowing due the impact and interconnection of dysphagia and tracheostomies (McRae et al, 2020).

Research in the field of conducting dysphagia assessment in adults with a tracheostomy is well reported (Brodsky et al., 2019). However, despite recommendations in the literature for intervention post assessment, Brodsky and colleagues (2019) note the lack of scientific based consensus for when and how intervention can begin post tracheostomy placement. Regardless of tracheostomy status, dysphagia assessment aims to support an intervention programme that is goal centred such as minimising the risk of aspiration and increase quality of life (Brodsky et al, 2019). In their scoping review, Skoretz and colleagues (2020) investigated swallowing and tracheostomy following critical illness. Based on their protocol they were able to include 85 appropriate studies of which $25 \%(n=21)$ of the papers looked at interventions. In the results the authors provided an overview of intervention type. They used the term "intervention" broadly to refer to instrumentation, traditional dysphagia exercises and manipulation of tracheostomy (e.g. deflation of the cuff and use of one-way valve to restore subglottic pressures required for swallowing). They noted that compared to the other domains of their research content (e.g. dysphagia frequency) there were relatively fewer studies available and thus acknowledge paucity in the literature regarding intervention for adults with dysphagia and tracheostomies.

Intervention, rehabilitation, management and exercise are some of the terms used to describe oropharyngeal dysphagia therapy. Therapy is a multimodal approach which aims to restore swallow function (Crary \& Carnaby, 2014; Smithard, 2016). Examples may include 'effortful swallowing' targeting the contact between base of tongue and posterior pharyngeal wall (Logemann, 1991); 'Mendelsohn manoeuvre' for laryngeal elevation (Mendelsohn et al, 1987) and the 'head lift' to improve hyoid displacement (Shaker et al., 1997). Compensatory strategies may include adaptation of bolus delivery 
and/or environmental factors (e.g. posture/position) (Brodsky et al., 2019). In patients with progressive neurological disorders gains were seen in functional swallowing tasks with oral trials and swallowing related quality of life measures (Athukorala et al., 2014). Behavioural interventions can lead to improved swallowing ability, reduced number of patients with dysphagia at the end of the trial, and had greater outcomes than other interventions such as acupuncture, electrical stimulation and drug therapies (Bath, et al, 2018). Technological advances have delivered new approaches as well as interventional tools to aid delivery of behavioural interventions e.g. surface electromyography to provide biofeedback (Archer et al., 2020). In recent years, neuromuscular electrical stimulation (NMES)/transcutaneous electrical stimulation (TES) and pharyngeal electrical stimulation (PES) have gained prominence as dysphagia therapeutic interventions (Dziewas, et al, 2018). These methods use electrodes that are either placed externally on submental muscles (NMES/TES) or internally via electrodes on a catheter along the posterior pharyngeal wall (PES) to provide electrical stimulation which results in musculature and/or cortical changes (Ludlow, et al, 2010; Vasant et al, 2016).

Dysphagia therapies have been evaluated and scrutinized in differing patient cohorts including but not limited to, stroke, Parkinson's disease, respiratory conditions and a variety of head and neck cancers. Dysphagia therapy is an accepted means of supporting patients to return to oral intake. Functional outcomes (e.g. increasing oral intake) after a programme of swallowing therapy have been noted on repeat videofluoroscopy assessments (Huang et al., 2014; Kang et al., 2012). Despite these gains, the evidence supporting the use of dysphagia therapy in patients with a tracheostomy is limited. One possible reason for this could be that developing a therapeutic protocol in this patient cohort is challenging due to the complex interaction of dysphagia and tracheostomies.

Swallowing in patients that have a tracheostomy has been considered a fundamentally important role in patient outcomes (NCEPOD, 2014). From Skoretz and colleagues' (2020) scoping review it is clear that therapeutic intervention is an area that needs further exploration. No published scoping review has extrapolated information secondary to dysphagia therapy in tracheostomised patients when considering treatment type, dosage and frequency. This detailed understanding of the scope and effectiveness of dysphagia therapy in this population cohort will help develop the evidence base, provide scientific knowledge to work towards a consensus in dysphagia therapy for people with a tracheostomy and support robust therapeutic trials in this population.

This scoping review aims to:

- Describe the existing published literature on oropharyngeal dysphagia therapy used in an adult population that have a tracheostomy at the time of reporting.

\section{Developing the research question:}

There is paucity in the literature regarding swallowing therapy in adults that have a tracheostomy (Skoretz et al, 2020). Further understanding of this area, including therapy type and dosage, can provide 
support to clinicians working with patients that have a tracheostomy and guide robust research in this area.

The research question posed is: What is known from the existing literature about dysphagia therapy that people with a tracheostomy are receiving. This question was used to conduct a literature search to gain up to date information about treatment methods available in adult populations. This protocol is specifically interested in participants with a tracheostomy that receive dysphagia therapy including: surgical, pharmaceutical, cortical and peripheral stimulation, alternative therapies and direct oropharyngeal exercises.

Manipulation of the tracheostomy including taking the cuff down and placing a speaking valve are synonymous with dysphagia therapy. This protocol agrees with these sentiments, however a thorough scoping review has recently been completed which minimises the knowledge gap in this area (Skoretz, Anger, Wellman et al, 2020) and therefore inclusion within this protocol is not required.

\section{Method / Design}

A scoping review methodology was chosen to describe the current available literature on the study topic. Due to the anticipated limited search yield, a broad approach to review the data is beneficial to map the literature (Peters, 2020). This will allow the authors to review the range of protocols and methodologies utilised by different researchers in a similar area which can be used to guide discussions into therapy options for adults in the future.

The design of this scoping review protocol uses the Joanna Briggs Institute (JBI) guidelines to ensure a systematic approach and facilitate repeatable measures (Aromataris \& Munn, 2020). It will follow the five stages included when conducting a scoping review as outlined by Arksey \& O'Malley, 2005. A PRISMAScR checklist will record the papers found and the subsequent numbers of included / excluded papers (see appendix 1).

\section{Eligibility Criteria}

Inclusion Criteria

1. Adults ( $\geq 18$ year old)

2. Participants assessed as having an oropharyngeal dysphagia but not patient reported dysphagia

3. Participants with a tracheostomy at the time of the study

4. The implementation of oropharyngeal therapy alone or in combination with tracheostomy manipulation. Examples of therapy intervention may include but not limited to respiratory muscle training, oro-musculature strength training, electrotherapeutic interventions

5. Participants location: inpatient (acute care hospital and/or acute care rehabilitation facilities) or receiving input whilst in the community i.e. their place of residence, outpatient clinics 
6. If a proportion of the patient sample in a study meet our inclusion criteria, where possible, we will extract individual patient-level data for only those meeting our inclusion criteria.

\section{Exclusion Criteria}

1. Participants $<18$ years old

2. Participants that have had oesophageal surgery or head and/or neck cancer diagnoses

3. Swallowing therapy protocols that are not clearly defined or discernible for patients with a tracheostomy

4. Studies that only discuss tracheostomy manipulation (e.g. cuff down and speaking valve) as a method of therapeutic intervention

\section{Information Sources:}

The scoping review design enables researchers to collate data from multiple sources (Peters, 2020). To ensure the robustness of the data being reviewed, the following inclusion and exclusion criteria will be applied to the type of information sources obtained in this review.

\section{Inclusion:}

- Case reports, case series, experimental studies, randomised control trials, observational studies and systematic reviews.

- Due to the anticipated limited search results, commentaries, opinion pieces, quality assurance and service delivery assessment studies will be included.

- The search period is from the start of online availability (database specific) to July 2021 inclusive

\section{Exclusion:}

- Articles not written or available in English;

Where a full text article cannot be obtained using University access, the 1 st author will be contacted. If no English copy is made available prior to final analysis, then the paper will be excluded.

- Articles will need to report sufficient treatment information including treatment type, dosage and intensity. Articles without sufficient protocol information as outlined will be excluded.

\section{Databases to be searched}

Initial searches will be conducted via the following electronic databases (Table 1): 
Table 1

Electronic databases

\begin{tabular}{|ll|}
\hline Platform & Database \\
\hline EBSCOhost & Medline \\
& PubMed \\
& CINAHL \\
OVID online & Cochrane Database of systematic reviews \\
& AMED \\
& SCOPUS \\
& Emcare \\
& LLBA \\
& Psyclnfo \\
& Web of Science Core Collection \\
\hline
\end{tabular}

Grey literature repositories via social media, open access thesis, conference proceeding abstracts, dissertations, Google Scholar, ResearchGate or from clinical experts in this field will also be considered if they meet the outlined inclusion/exclusion criteria.

This strategy was then peer reviewed by two collaborators.

\section{Search Strategy:}

The search strategy was developed with Breege Whiten (BW), University College London librarian. The strategy is in two stages to ensure that all relevant articles can be included in this study. The first stage will involve using the key search terms, created with BW, and applying them to the medical subject heading (MeSH)/keyword search where applicable (Table 2). A record of the number of articles found on each database will be made.

At the second stage, for the accepted articles we will perform forward and backward citation chasing (Booth, 2008). This will involve reviewing study titles citing the accepted articles for possible inclusion as well as their respective reference lists (Booth, 2008). We will then apply the eligibility criteria to these citations. 
Table 2

Key search terms used in the databases

\begin{tabular}{|lll|}
\hline $\begin{array}{l}\text { Level } \\
\mathbf{1}\end{array}$ & Tracheostomy & Tracheo* \\
\hline $\begin{array}{l}\text { Level } \\
\mathbf{3}\end{array}$ & Therapy & $\begin{array}{l}\text { "Oropharyngeal therap*" OR "dysphagia therap*" OR therap* OR } \\
\text { "Oropharyngeal rehab*" OR rehab* OR treatment* OR intervention* OR } \\
\text { exercise* }\end{array}$ \\
\hline $\begin{array}{l}\text { Level } \\
4\end{array}$ & Adults & $\begin{array}{l}\text { Adult OR aged OR elderly OR middle-age* } \\
5\end{array}$ \\
\hline $\begin{array}{l}\text { Level } \\
5\end{array}$ & Setting & Inpatient* OR outpatient* OR clinic \\
\hline
\end{tabular}

\section{Study Selection}

1. The primary reviewer (WK) will run the initial searches and export the titles and abstracts into and online platform such as www.covidence.org.

2. Duplicate copies will be deleted.

3. Two reviewers (WK and SM) blinded to each other's judgements will screen the title and abstracts of each paper for inclusion or exclusion.

4. A record of decisions will be kept on the online platform.

5. Full text for accepted abstracts will be retrieved.

Similar to Skoretz et al (2020) scoping review, if the accepted abstract existed only as such, the primary reviewer will contact the abstract authors to determine if its content existed either as: 1) a study published under a different name, 2) grey literature, or 3 ) a raw data set (not available via any grey literature repository). If full text is not available, the abstract will not be put through to the next review phase.

Similar to Skoretz et al (2020) scoping review, if the accepted abstract existed only as such, the primary reviewer will contact the abstract authors to determine if its content existed either as: 1) a study published under a different name, 2) grey literature, or 3) a raw data set (not available via any grey literature repository). If full text is not available, the abstract will not be put through to the next review phase.

The full text reviews will be conducted to determine final eligibility, by two authors blinded to each other's judgments (WK, SM).

6. A study selection form will be designed and used throughout the process.

7. At both the abstract and full text review any conflicts will be discussed and clarified. If conflicts cannot be resolved a third author $(\mathrm{RH})$ will be asked to resolve the conflict. If all reviewers remain undecided regarding the suitability for inclusion following abstract review, the citation will be accepted and full text will be reviewed 


\section{Data extraction and Analysis}

Following literature retrieval data will be extracted by two authors blinded to the others judgement (WK and SM).

The data extraction will be placed in an excel database and will include information as suggested by Hofman and colleauges (2014) from their article: Better reporting of interventions: template for intervention description and replication (TIDieR) checklist and guide. For instance:

- Title

- Authors

- Methodology

- Participant demographics

- Study population/aetiology

- Outcome measures.

- Details of therapy will also be extracted specifically:

type of exercises, dosage, intensity, format and comparator where available.

The two reviewers (WK and SM) will extract available data including design, sample size, how swallowing assessment was conducted, data related to the tracheostomy and in particular if this was discussed in relation to therapeutic programme.

This data will be reviewed and analysed against the outlined inclusion / exclusion criteria by two reviewers (WK and SM). If there is a disagreement a third reviewer $(\mathrm{RH})$ will resolve conflicts. www.covidence.org online software will be used to record the final decisions.

\section{Results}

\section{Data Presentation and Dissemination:}

As per scoping review protocols, a flow diagram will be used to demonstrate data extraction. The data extracted from each article will be presented in written and visual format. Depending on the quantity of papers extracted, where possible the data will be separated into aetiologies to draw conclusions regarding therapy designed for certain aetiologies.

Identification of therapeutic techniques used with adults that have a tracheostomy will be discussed including potential benefits and limitations. The type of review paper will be provided along with specified outcome measures. The study design will be analysed to support replicability of therapeutic techniques in specific aetiologies of patients with a tracheostomy. 


\section{Discussion}

The aim of this scoping review is to answer the research question by gathering empirical evidence about dysphagia therapy in adults that have a tracheostomy. This information will inform future studies in creating robust consensus on dysphagia therapy programmes with clear outcome measures. The sparsity in the literature in this field supports the notion of further research in this area to fully understand the impact of dysphagia therapy in this population cohort.

Limitations to this protocol include the exclusion of non-English studies heightening the potential for language and cultural bias which will contribute to the anticipated limited studies included in the final study. If funding became available to translate studies, then this could be considered for any papers excluded based on this criteria and may provide additional information to this study's findings. The decision to include all aetiologies reduces the potential to provide specific dysphagia therapy recommendations to a specific population e.g. stroke. However, it is has already been recognised by Skoretz and colleagues (2020) that there is limited available literature in this area. Moreover, the aim of this protocol is to extrapolate detailed information pertaining to dysphagia therapy in adults with a tracheostomy. By doing so, it will hopefully enable future research to tease apart therapy for specific aetiologies, if felt clinically relevant.

\section{Declarations}

Ethics approval and consent to participate.

Not applicable for the protocol stage.

Consent for publication

Not applicable.

Availability of supporting data

Competing interests

The authors declare that they have no competing interests.

\section{Funding}

WK is funded by a National Institute for Health Research (NIHR), Pre-Doctoral Clinical Academic Fellowship. Grant reference: NIHR301122.

This paper presents independent research funded by the National Institute for Health Research (NIHR). The views expressed are those of the author(s) and not necessarily those of the NHS, the NIHR or the Department of Health and Social Care. 
Author Contributions

WK conceived the review and wrote the first drafts of the manuscript. $\mathrm{RH}, \mathrm{CS}, \mathrm{BW}$ and SM all participated in discussions regarding the methods and protocol design and reviewed subsequent drafts. CS, RH and BW provided minor revision which contributed to the final version. All authors read and approved the final manuscript.

\section{Acknowledgements}

The authors would like to acknowledge Natalie Griffın, Principal Speech and Language Therapist (Acute and Stroke), London North West University Healthcare NHS Trust for their support and clinical knowledge.

\section{Authors' information}

WK is a Highly Specialist Speech and Language Therapist at Northwick Park Hospital and an NIHR predoctoral clinical academic fellow. WK is completing an MRes at University College London (UCL) in the division of Psychology and Language Sciences.

$\mathrm{RH}$ is a Highly Specialist Speech and Language Therapist at Great Ormond Street Hospital and an NIHR predoctoral clinical academic fellow. She is completing an MRes at University College London (UCL) in the division of Psychology and Language Sciences.

BW, Librarian at UCL Language \& Speech Science Library, University College London.

CS is an Associate Professor within the division of Psychology and Language Sciences at University College London. Research interests include speech and swallowing in adults and in children populations

SM is Acting Clinical Lead Speech and Language Therapist (Adult Acute Service), Addenbrookes Hospital, Cambridge University Hospitals NHS Foundation Trust.

\section{Abbreviations}

JBI - Joanna Briggs Institute

MeSH - medical subject headings

PRISMA - Preferred Reporting Items for Systematic Reviews and Meta-Analyses

PRISMA-ScR - Preferred Reporting Items for Systematic Reviews and Meta-Analyses - Scoping Reviews

Rayyan QCRI - Rayyan Qatar Computing Research Institute

RCSLT - Royal College of speech and language therapists

sEMG - surface electromyography 
UCL - University College London

UK - United Kingdom

\section{References}

1. Archer, S.K., Smith, C.H. \& Newham, D.J. (2020). Surface Electromyographic Biofeedback and the Effortful Swallow Exercise for Stroke-Related Dysphagia and in Healthy Ageing. Dysphagia. https://doi.org/10.1007/s00455-020-10129-8

2. Arksey, H. \& O'Malley, L. (2005). Scoping studies: towards a methodological framework. International Journal of Social Research Methodology. 8:1.doi:10.1080/1364557032000119616

3. Aromataris E, Munn Z (Editors). JBI Manual for Evidence Synthesis. JBI, 2020. Available from https://synthesismanual.jbi.global. https://doi.org/10.46658/JBIMES-20-01

4. Athukorala R, Jones R, Sella O, Huckabee M. Skill training for swallowing rehabilitation in patients with parkinson's disease. Archives of Physical Medicine and Rehabilitation. Elsevier Ltd. 2014;95:7. doi: 10.1016/j.apmr.2014.03.001

5. Bath, P., Lee, H. and Everton, L. (2018). Swallowing therapy for dysphagia in acute and subacute stroke. Cochrane Database of Systematic Reviews.

6. Booth A: Unpacking your literature search toolbox: On search styles and tactics. Health Info Libr J $2008 ; 25: 313-317$

7. Bonanno, P. C., (1971). Swallowing dysfunction after tracheostomy. Annals of Surgery, 174(1), 2933.

8. Brodsky MB, Mayfield EB, Gross RD. (2019). Clinical Decision Making in the ICU: Dysphagia Screening, Assessment, and Treatment. Semin Speech Lang. 40(3):170-187. doi: 10.1055/s-00391688980. PMID: 31158902.

9. Cheung NH, Napolitano LM. (2014). Tracheostomy: epidemiology, indications, timing, technique, and outcomes. Respir Care. 59(6):895-915; discussion 916-9. doi: 10.4187/respcare.02971. PMID: 24891198.

10. Crary, M. and Carnaby, G. (2014). Adoption into clinical practice of two therapies to manage swallowing disorders. Current Opinion in Otolaryngology \& Head and Neck Surgery, 22(3), pp.172180.

11. Dziewas, Rainer, Stellato, Rebecca, Van der Tweel, Ingeborg, Walther, Ernst, Werner, Cornelius J, Braun, Tobias, ... Pfausler, Bettina. (2018). Pharyngeal electrical stimulation for early decannulation 
in tracheotomised patients with neurogenic dysphagia after stroke (PHAST-TRAC): A prospective, single-blinded, randomised trial. Lancet Neurology,17(10), 849-859.

12. Hofman T, Glasziou P, Boutron I, Milne R, Perera R, Moher D, Altman D, Barbour V, Macdonald H, Johnston M, Lamb S, Dixon-Woods M, McCullough P, Wyatt J, Chan A, Michie S (2014) Better reporting of interventions: template for intervention description and replication (TIDieR) checklist and guide. BMJ 348:1687

13. Huang, K., Liu, T., Huang, Y., Leong, C., Lin, W. and Pong, Y. (2014). Functional Outcome in Acute Stroke Patients with Oropharyngeal Dysphagia after Swallowing Therapy. Journal of Stroke and Cerebrovascular Diseases, 23(10), pp.2547-2553.

14. Leder, S. B. and Ross, D. A., 2000, Investigation of the causal relationship between tracheotomy and aspiration in the acute care setting. Laryngoscope.110(4), 641-644.

15. Leder, S.B. \& Ross, D.A. 2010. Confirmation of no causal relationship between tracheotomy and aspiration status: a direct replication study. Dysphagia. 25, (1) 35-39

16. Logemann JA. (1991). Approaches to management of disordered swallowing. Bailliere's Clinical Gastroenterology. 5:2. doi: 10.1016/0950-3528(91)90030-5

17. Ludlow, C L. (2010). Electrical neuromuscular stimulation in dysphagia: current status, Current Opinion. Otolaryngology \& Head and Neck Surgery. 18(3) 159-164 doi: $10.1097 / \mathrm{MOO} .0 \mathrm{~b} 013 \mathrm{e} 3283395 \mathrm{dec}$

18. Kang, J., Park, R., Lee, S., Kim, J., Yoon, S. and Jung, K. (2012). The Effect of Bedside Exercise Program on Stroke Patients with Dysphagia. Annals of Rehabilitation Medicine, 36(4), p.512.

19. Marik, PE. (2001). Aspiration pneumonitis and aspiration pneumonia. New England Journal of Medicine, 344(9), 665-671.

20. McRae, J., Montgomery, E., Garstang, Z., \& Cleary, E. (2020). The role of speech and language therapists in the intensive care unit. Journal of the Intensive Care Society, 21(4), 344-348. https://doi.org/10.1177/1751143719875687

21. Mendelsohn MS, McConnel FM. (1987). Function in the pharyngoesophageal segment. Laryngoscope. 97:4. doi: 10.1288/00005537-198704000-00014.

22. NATIONAL CONFIDENTIAL ENQUIRY PATIENT OUTCOME AND DEATH (NCEPOD). (2014). On the Right Trach? A Review of the Care Received by Patients Who Underwent a Tracheostomy (available at: http://www.ncepod.org.uk/2014report1/ downloads/On\%20the\%20Right\%20Trach_FullReport.pdf) (accessed on 7th January 2021). 
23. Peters, MDJ., Marnie, C., Tricco AC., Pollock, D., Munn, Z., Alexander, L., Mclnerney, P., Godfrey, M., Khalil, H. Updated methodological guidance for the conduct of scoping reviews. JBI Evidence Synthesis. 2020;18:10. doi:10.11124/JBIES-20-00167

24. Skoretz, SA., Anger, N., Wellman, L., Takai, O., \& Empey, A. (2020). A Systematic Review of Tracheostomy Modifications and Swallowing in Adults. Dysphagia,35(6), 935-947.

25. Skoretz, SA., Riopelle, SJ., Wellman, L., \& Dawson, C. (2020). Investigating Swallowing and Tracheostomy Following Critical Illness: A Scoping Review. Critical Care Medicine. 48-2. doi: 10.1097/CCM.0000000000004098

26. Shaker R, et al. (1997). Augmentation of deglutitive upper esophageal sphincter opening in the elderly by exercise. American Journal of Physiology - Gastrointestinal and Liver Physiology. 272:6. doi: 10.1152/ajpgi.1997.272.6.g1518

27. Smithard, D. (2016). Dysphagia Management and Stroke Units. Current Physical Medicine and Rehabilitation Reports, 4(4), pp.287-294.

28. Vasant, D. H., Michou, E., O'Leary, N., Vail, A., Mistry, S., \& Hamdy, S. (2016). Pharyngeal Electrical Stimulation in Dysphagia Poststroke: A Prospective, Randomized Single-Blinded Interventional Study. Neurorehabilitation and Neural Repair, 30(9), 866https://doi.org/10.1177/1545968316639129

29. Wallace, S., McGowan, S. and Ginnelly, A., 2016. Oral feeding in tracheostomy patients- The Royal College of Speech and Language Therapists tracheostomy clinical excellence network responds. Journal of the Intensive Care Society, 17(2), 180

30. Walshe, M.(2019) Swallowing and ageing, Speech, Language and Hearing, 22:1, 28, DOI: 1080/2050571X.2019.1567898

\section{Supplementary Files}

This is a list of supplementary files associated with this preprint. Click to download.

- AppendixAPRISMAScRFillableChecklist.docx 